\title{
A Novel Road Extraction Algorithm for High Resolution Remote Sensing Images
}

\author{
Teng Xinpeng, Song Shunlin* and Zhan Yongzhao \\ School of Computer Science and Telecommunication Engineering, Zhenjiang, Jiangsu, 212013, China
}

Received: 2 Jul. 2013, Revised: 6 Nov. 2013, Accepted: 7 Nov. 2013

Published online: 1 May. 2014

\begin{abstract}
Automatic road extraction from the high resolution remote sensing images is of great importance in intelligent transportation and image processing. Hence, in this paper, an effective road extraction algorithm for high resolution remote sensing images based on the circular projection transformation is proposed. The main idea of the proposed algorithm lies in that the road extraction results are obtained by selecting a suitable initial template, and then search the matched templates through moving the initial template in two directions. Firstly, the circular projection vector of the initial template is achieved by calculating the circular projection value at a specific radius. Secondly, the optimal radius of the circle in circular projection transformation and the length of the seeking step and the seeking angle are determined. Thirdly, for each seeking step the similarity between the target template and the initial template is computed, and the template with the highest similarity is chosen. Finally, roads can be detected by the correct direction by exchanging the first two detected points. To make performance evaluation, the IKONOS dataset is utilized and DMES and AUA algorithm are compared. The experimental results demonstrate that the proposed algorithm can automatic the roads from high resolution remote sensing images effectively and efficiently.
\end{abstract}

Keywords: Road extraction, Remote sensing images, Circular projection transformation, Template, ROC curve

\section{Introduction}

As is shown in Wikipedia, remote sensing refers to the acquisition of information about an object or phenomenon without making physical contact with the object. In modern usage, this term generally means the application of aerial sensor technologies to detect and classify objects on Earth through the propagated signals. Particularly, there are two main kinds of remote sensing, which are 1) passive remote sensing and 2) active remote sensing. The first kind of sensors can detect natural radiation which is emitted or reflected by the object or surrounding areas. The second kinds of sensors could emit energy to scan objects and areas whereupon a sensor then detects and measures the radiation that is reflected or backscattered from the target. Furthermore, remote sensing can collect data on dangerous or human inaccessible areas. Remote sensing applications consist of monitoring deforestation in several areas $[1,2]$. Remote sensing images are obtained by multispectral sensors, such as the widely used Landsat Thematic Mapper sensor. Particularly remote sensing images are widely used in numerous earth observation related applications [1,3].
Remote sensing is of great importance to be used in collecting geographical data and huge remote sensing images have been increasing rapidly. Currently, thousands of GB remote sensing images can be generated and need to be managed. The high resolution remote sensing images are vital for the fields of national defense, disaster relief and so on [4]. However, the massive remote sensing images are stored and managed in a distributed mode. Hence, it is difficult to organize and utilize these remote sensing images effectively. Particularly, the geographical objects in remote sensing images can not be retrieved effectively. If the objects can be detected accurately from remote sensing images, some important information can be extracted from remote sensing images [5]. For the high resolution remote sensing images, the road is an important kind of object. Moreover, road extraction from remote sensing images is a critical task in image processing. The reason lies in that recognition of roads is a key problem since they form an important GIS layer in significant civilian and military applications including navigation or location aware systems and emergency planning systems for evacuation and fire response $[6,7,8$,

\footnotetext{
*Corresponding author e-mail: 86265029@qq.com
} 
9]. Automatic road extraction algorithm can reduce both time and labor to construct and update the road spatial database in such applications. However, fully automated algorithms to recognize them for applications where accuracy is critical are very difficult $[6,10,11]$. In this paper, we utilize the high resolution remote sensing images, which can obviously enhance the object recognition accuracy.

The rest of the paper is organized as the following sections. Section 2 introduces the related works. In section 3 , overview of the road extraction problem using circular projection is given. Section 4 illustrates the proposed road extraction algorithm for high resolution remote sensing images. In section 5, experiments and related analysis are conducted to make performance evaluation. Finally, we conclude the whole paper in section 6 .

\section{Related works}

As road extraction is of great importance in image process, there are many researchers who have began to be engaged in this field. In this section, we will illustrate the traditional methods of road extraction.

Zhang et al. proposed a multilevel information fusion approach by combining the fuzzy clustering-based feature level information fusion (FCMA) and the modified Dempster-Shafer evidence reasoning-based decision level information fusion (D-SEMA). The FCMA can extract the key features from atomic messages, thereby greatly reducing the network traffic load. Furthermore, the D-SEMA mechanism is utilized to judge whether the road congestion event occurs [12].

Jiang et al. described a novel navigation path detection algorithm in an unstructured environment. Natural frames are analyzed in RGB vector space to research the feasibility of curve path detection of unstructured roads. Perceptual color clustering and morphological image processing have been used as pre-processing to obtain the segmented path. The pixels of the segmented image are analyzed from right to left line-by-line to find the middle points of the path. In order to obtain the optimal navigation path, the least-squares curve fitting method has been used in the proposed research [13].

Demissie et al. explored a complementary method to gauge the status of road traffic conditions through the use of cellular networks handover count. To test this method, hourly handover counts were obtained in Lisbon, Portugal, from 39 cellular towers in the vicinity of arterial roads that have 12 traffic counters with an average daily traffic size of 20,500 vehicles. An initial correlation analysis proved the existence of a good relationship between handover and traffic volumes. Furthermore, the authors classified the hourly traffic counts into three categories: high, medium, and low traffic levels using the 50 th and 80 th percentiles. Then, half of the data was used to build a multinomial logit (MNL) model and to train an artificial neural network (ANN) in order to relate traffic and handover [14].

Siogkas et al. proposed a new fully automatic algorithm that combines both time and spatial information using the efficient random-walker algorithm (RWA) as a segmentation tool. Particularly, a novel technique for automatic seed selection is proposed, utilizing features derived from a shadow-resistant optical flow estimator using the $c(1)$ channel of the $c(1) c(2) c(3)$ color space, along with a priori information and previous frame segmentation results [15].

Shang et al. $\mathrm{p}$ roposed a novel algorithm which combines SVM, KNN and the confidence map under a Bayesian framework to improve the overall performance of the unstructured road detections. The proposed algorithm has been evaluated using different types of unstructured roads and the experimental results show its effectiveness [16].

Oliveira et al. proposed a fully integrated system for the automatic detection and characterization of cracks in road flexible pavement surfaces to minimize the human subjectivity resulting from traditional visual surveys. The first task addressed, i.e., crack detection, is based on a learning from samples paradigm, where a subset of the available image database is automatically selected and used for unsupervised training of the system. The system classifies nonoverlapping image blocks as either containing crack pixels or not. The second task deals with crack type characterization, for which another classification system is constructed, to characterize the detected cracks' connect components [17].

Cheng et al. presented a road junction extraction method with two stages. First, global detection is performed to find the centre positions of the road junction candidates by using morphological operators. Second, the shape of a road junction is identified based on a valley-finding algorithm. The proposed method is validated by airborne synthetic aperture radar (SAR) images of $1 \mathrm{~m}$ resolution [18].

Unsalan et al. proposed a novel system, which has three main modules: 1) probabilistic road center detection, 2) road shape extraction, and 3) graph-theory-based road network formation. These modules may be used sequentially or interchangeably depending on the application at hand. To show the strengths and weaknesses of the proposed system, the authors tested it on several very high resolution satellite (Geoeye, Ikonos, and QuickBird) and aerial image sets [19].

Guo et al. presented a robust stereo-vision-based drivable road detection and tracking system that was designed to navigate an intelligent vehicle through challenging traffic scenarios and increment road safety in such scenarios with advanced driver-assistance systems (ADAS). This system is based on a formulation of stereo with homography as a maximum a posteriori (MAP) problem in a Markov random field (MRF). Under this 
formulation, The authors developed an alternating optimization algorithm that alternates between computing the binary labeling for road/nonroad classification and learning the optimal parameters from the current input stereo pair itself [20].

The following research is quite similar to ours, however, our proposed algorithm is more suitable to the high resolution remote sensing images.

Chaudhuri et al. presented a semi-automatic approach for road detection that achieves high accuracy and efficiency. This proposed method exploited the properties of road segments to develop customized operators to accurately derive the road segments. The customized operators include directional morphological enhancement, directional segmentation and thinning [6].

Different from the above papers, in this work, we study on how to effectively seek the roads from high resolution remote sensing images by the circular projection transformation.

\section{Illustration of the road extraction problem using circular projection}

In this paper, we propose a novel road extracting algorithm by the circular projection transformation. Particularly, the roads in high resolution remote sensing images can be detected by circular projection through expanding the circles. As is shown in Fig.1, fragment of a remote sensing image is given, and then the road with a branch is detected. Particularly, the road is represented by red line. In the following sub-section, the theory of circular projection transformation will be described in detail.

Supposing $S$ denotes a $N \times N$ reference graph(Fig.2(a)), $\mathrm{T}$ represents a $M \times M$ template graph(Fig.2(b)), and $S^{i, j}$ means a searching $\operatorname{sub}-\operatorname{graph}(i, j \in[0, N-M])$ which is covered by the template graph.

Afterwards, image $T$ is represented by polar coordinates, and the central point(denoted as $o$ ) of image $T$ is represented as origin of coordinates(Shown in Fig.2(c)). Hence, the polar coordinate system could be built successfully. Assuming the coordinate of point $o$ is $(\varphi, \phi)$, the circular projection vector $P(r)$ is defined as follows.

$$
P(r)=\sum_{\theta=0}^{2 \pi} T(r, \theta), r \in[0, R]
$$

where $r=\sqrt{(m-\varphi)^{2}+(n-\phi)^{2}}$, and $R$ represents the radius of maximum inscribed circle in image $T$.

From the above, we can see that the scale of template graph is defined as $M \times M$, after conduct the circular

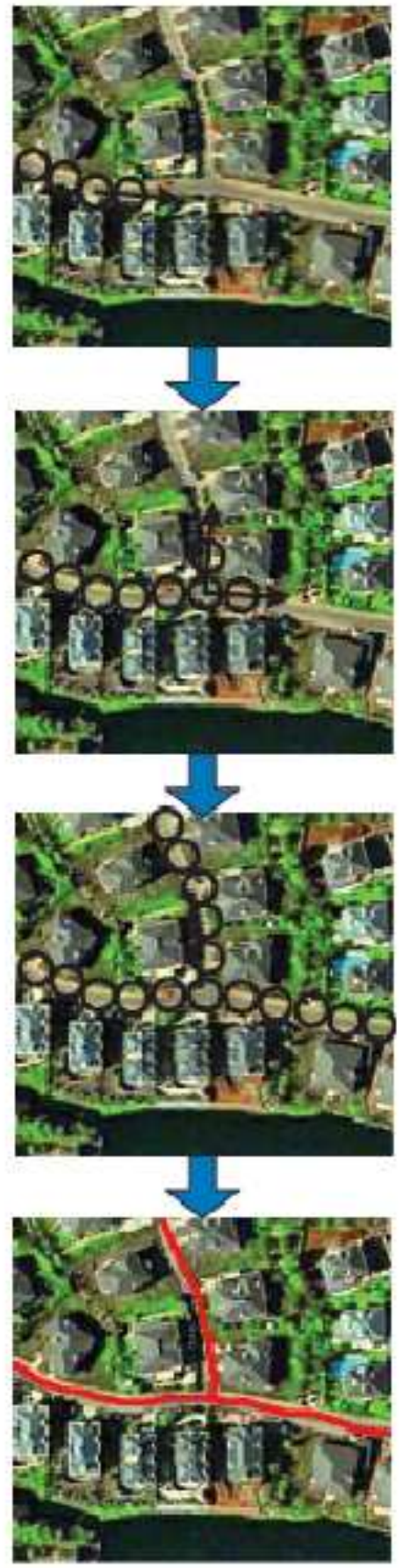

Fig. 1: An example of road extraction from high resolution remote sensing images

projection transformation, the following equation can be obtained.

$$
P_{T}(r)=\frac{\sum_{k} T\left(r \cdot \cos \theta_{k}, r \cdot \sin \theta_{k}\right)}{S_{r}}, \theta_{k} \in(0,2 \pi]
$$




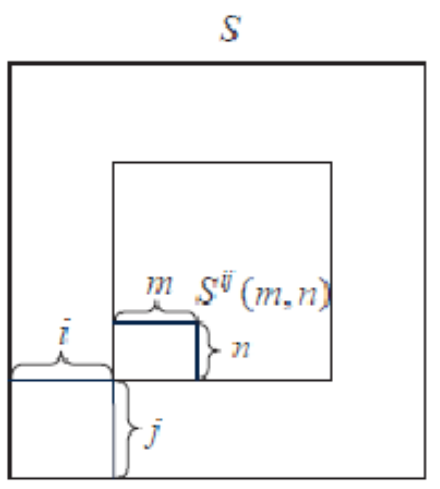

(a) Reference graph

$T$

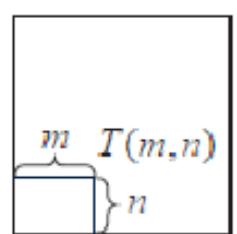

(b) Template graph

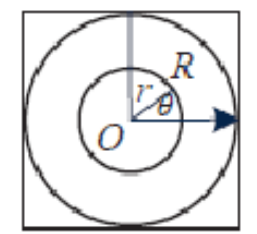

(c) Circular projection vector
Fig. 2: The illustration of circular projection process

where $S_{r}$ denotes the total number of pixels in the circle, and the radius of this circle is $r . T(x, y)$ represents the value of the pixel which is located at $(x, y)$. The central point $o$ with the coordinate $(\varphi, \phi)$ of the given template $T$ can be converted from a Cartesian coordinate to the polar coordinate by the following equation.

$$
\begin{aligned}
& \widehat{\varphi}=\varphi+r \cdot \cos \theta \\
& \widehat{\phi}=\phi+r \cdot \sin \theta
\end{aligned}
$$

Particularly, supposing the current template position in the remote sensing image is represented as $(x, y)$, the circular projection transformation value(denoted as $\left.P_{S}(x, y, r)\right)$ of the current sub-image at the radius $r$ is defined in the following equation.

$$
P_{S}(x, y, r)=\frac{\left.\sum_{k}^{I(} x+x_{c}+r \cos \theta_{k}, y+y_{c}+r \sin \theta_{k}\right)}{S_{r}}
$$

where $S_{r}$ refers to the total number of pixels located in the range of the circle, the radius of which is equal to $r$. The NC between the vectors of circular projection transformation of the template and the sub-image at the coordinate $(a, b)$ is represented as $\psi(a, b)$ as follows.

$$
\psi(a, b)=\frac{\eta(a, b)}{\left\|P_{S}(a, b)\right\| \cdot\left\|P_{T}\right\|}
$$

where $\eta(a, b)$ means the relationship between the value of the circular projection transformation and the current sub-image, and $\left\|P_{S}(a, b)\right\|$ means the L2-norm of the circular projection transformation vector. Particularly, $\eta(a, b)$ can be achieved as follows.

$$
\eta(a, b)=\sum_{r=0}^{R} P_{S}(a, b, r) \cdot P_{T}(r)
$$

\section{Road extraction algorithm utilizing circular projection transformation}

In this section, we will demonstrate the road extraction algorithm by the above proposed circular projection transformation. Actually, the ratio of road in the remote sensing image is quite small. Hence, it is considerably time-consuming to compute the vector of circular projection in the whole remote sensing image. Therefore, our proposed algorithm begins from a chosen template, and the road can be extracted by expanding the initial template. The main idea of our algorithm lies in that the road extraction process is implemented from the initial template, and then other matched template is seeking by moving the initial template in two directions. The proposed algorithm is illustrated as follows.

Algorithm 1: Road extraction algorithm utilizing circular projection transformation for high resolution remote sensing images

Input: High resolution remote sensing image, the initial template

Output: The roads detected

(1) Calculating the circular projection vector of the initial template by computing the circular projection value at radius $r$ (denoted as $c p v(r))$ corresponding to different radius.

$\operatorname{cpv}(r)=\frac{\sum_{\theta=0}^{2 \pi} I(r \cdot \cos \theta, r \cdot \sin \theta)}{2 \pi r}, r \in(0, R], \theta \in[0,2 \pi)$

(2) Determining the optimal radius of the circle in circular projection transformation

(3) Determining the length of the seeking step and the seeking angle.

(4) For each seeking step do

(5) Computing the related circular projection vector and the similarity of it and the initial template 

$\left(S_{k}\right)$

(6) Choosing the template with the highest similarity

$S_{k}=\frac{\sum_{i=1}^{C_{k}} I\left(x+\left\lfloor\frac{R}{N_{R}} \cdot k \cdot \cos \left(\frac{2 \cdot \pi \cdot i}{C_{k}}\right)\right\rfloor, y+\left\lfloor\frac{R}{N_{R}} \cdot k \cdot \sin \left(\frac{2 \cdot \pi \cdot i}{C_{k}}\right)\right\rfloor\right)}{C_{k}}$

$$
k \in\left\{1,2, \ldots \ldots, N_{R}\right\}
$$

(7) If $S_{k}<\xi$ then goto step 9 ( $\xi$ is a pre-defined threshold)

(8) Setting the current location to the central point of template

(9) Seeking the road through the opposite direction by exchanging the first two detected points, and goto step 3

(10) End For

\section{Experiment}

In this section, performance evaluation of the proposed road extraction algorithm is conducted. IKONOS panchromatic remote sensing images are utilized as experimental dataset, which is captured from the city of Ghent in Belgium. In this dataset, images are normalized into a standard GEO product with a 1-m pixel resolution. Particularly, five kinds of road are contained in this dataset, which are represented as R1, R2, R3, R4, R5shown in Fig.3). A lot of small image subsets have been chosen which is made up of straight examples of typical roads. Before demonstrating the experimental results, the performance evaluation metric should be introduced in advance.

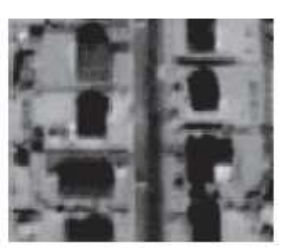

R1

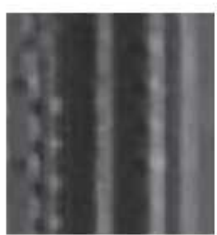

R3

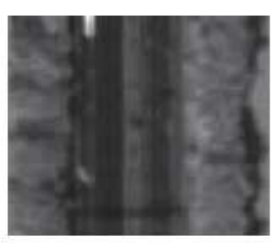

$\mathrm{R} 2$

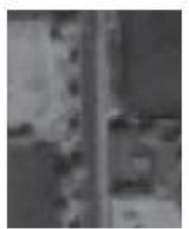

$\mathbb{R} 4$

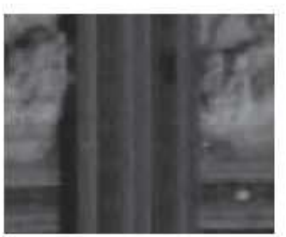

R5
Fig. 3: Examples of five kinds of road types in the IKONOS image dataset

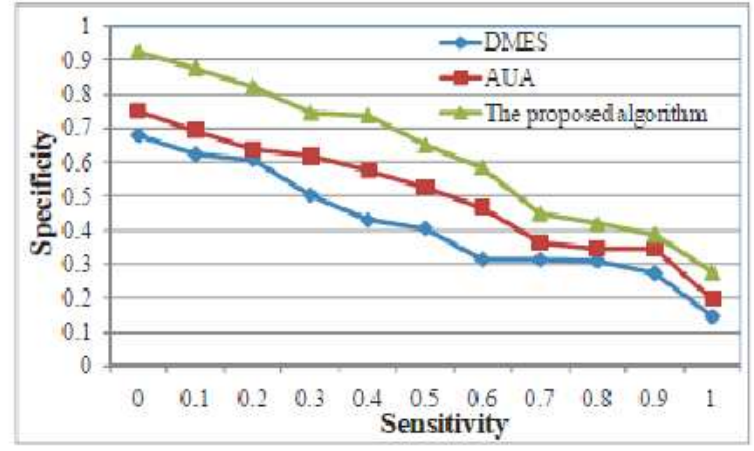

Fig. 4: ROC curves for specificity and sensitivity based on road type 1

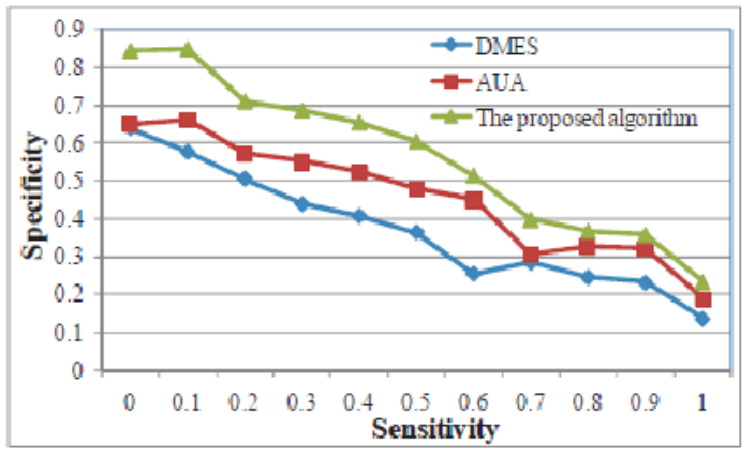

Fig. 5: ROC curves for specificity and sensitivity based on road type 2

In this experiment, Sensitivity and specificity are utilized as evaluation metric, which is defined as follows.

$$
\begin{aligned}
& \text { Sensitivity }=\frac{T P}{T P+F N} \\
& \text { Specificity }=\frac{T P}{T N+F P}
\end{aligned}
$$

where the parameters TP, TN, FP, FN refer to "true positive", "true negative", "false positive", and "false negative" respectively. Particularly, Sensitivity means the number of pixels in the detected road divided by the whole pixels in the road, and specificity denotes one subtracting the number of noise pixels in the detected road divided by the whole pixels in the road. To make performance comparison, other two methods are compared with the proposed method, which are 1) Directional Morphological Enhancement and Segmentation based road detecting method(DMES)[6], and 2) adaptive unsupervised approach for road 
detecting(AUA)[9]. Firstly, the ROC curves for specificity and sensitivity are illustrated in Fig.4-Fig.9.

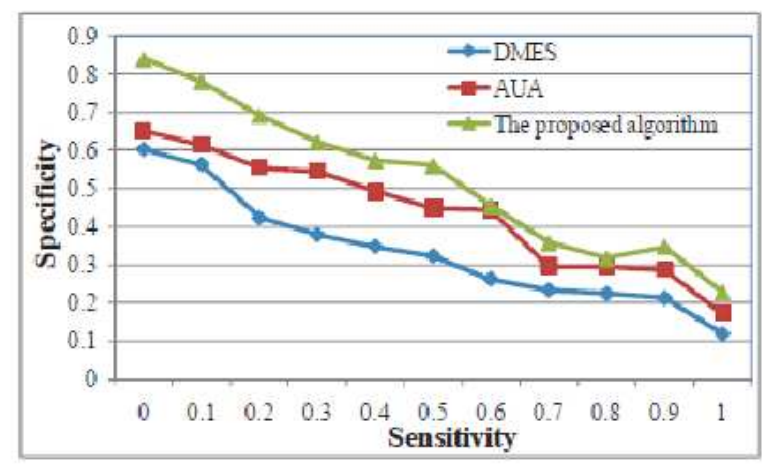

Fig. 6: ROC curves for specificity and sensitivity based on road type 3

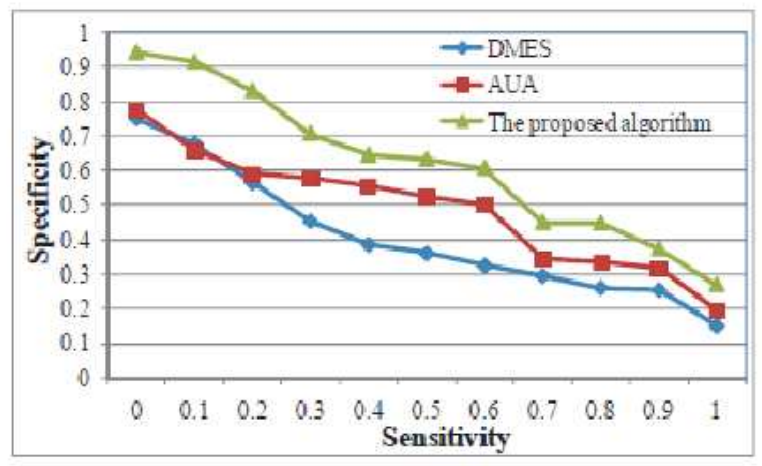

Fig. 7: ROC curves for specificity and sensitivity based on road type 4

As is shown in Fig.4-Fig.8, the ROC curve is applied, and the optimal performance for this curve is defined as the point on the curve closest to the upper right corner, the coordinate of which is equal to $r$. Furthermore, the upper right corner is corresponding to a specific road, which is effectively detected with no detection of noisy structures. It can be seen from Fig.4-Fig. 8 that the proposed performs better than DMES and AUA for all the five types of road. Secondly, road extraction precision for different road types are compared for different methods(shown in Fig.9). To implement this experiment, we utilize the roads detected by experts as the ground truth.

From Fig.4-Fig.9, we can know that the proposed algorithm can extract the roads more accurately than DMES and AUA, and the algorithm DMES performs

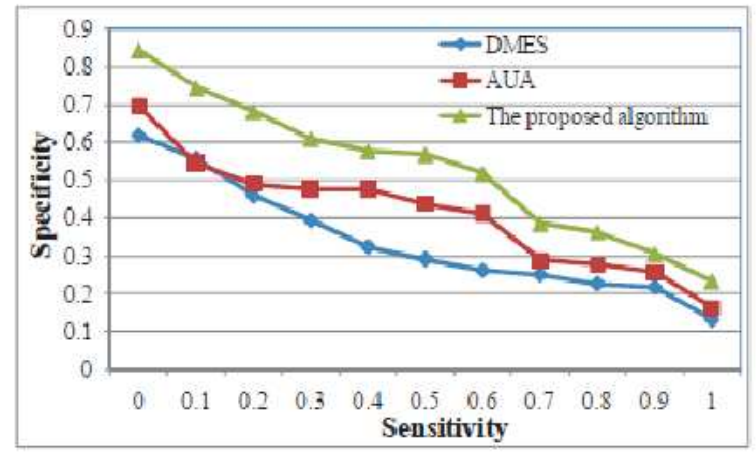

Fig. 8: ROC curves for specificity and sensitivity based on road type 5

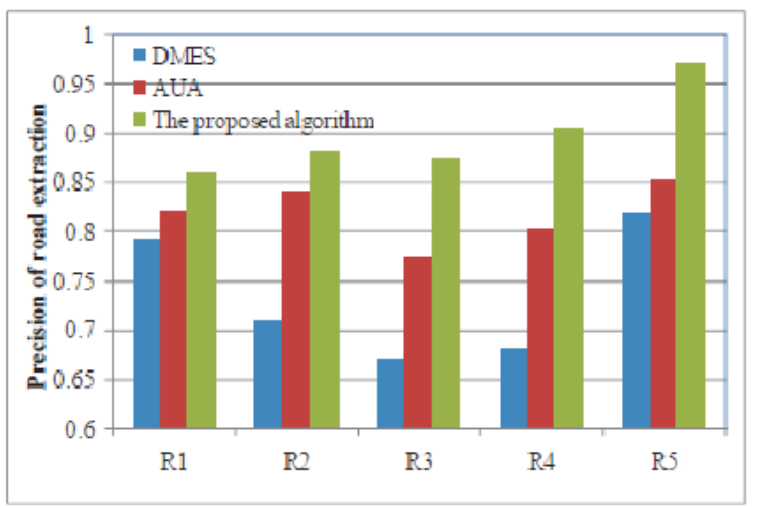

Fig. 9: Comparison of road extraction precision for different road types

better than AUA. The reason lies in the following aspects: 1) DMES utilizes both the spectral and spatial properties of roads using a multi-step method. The main steps in DMES include: "road enhancement", "road segmentation", "hole filling", "small region filtering", "length based region filtering", and "road segment linking". However, this approach does not consider the case that the roads in the image are incoherent or unclear. 2) AUA contains a novel two-step approach for automatic extraction of road networks from remote sensing images. The road detection step is based on shape classification of a local homogeneous region around a pixel. Particularly, the local homogeneous region is enclosed by a footprint of the pixel. However, the footprint classification based on Fourier shape description in AUA method should be improved to decrease the amount of leakage and finding a global connecting method which deals with the connections among road trees. 3) In the proposed algorithm, the road extraction results are obtained from 
the initial template, and then the matched templates are searched from moving the initial template in both directions.

In order to illustrate the performance of the proposed algorithm, some examples are given in Fig.10-Fig.13. In the above four figures, the roads extracted by the proposed algorithm are represented by the yellow lines, and the line width can reflect the road importance.

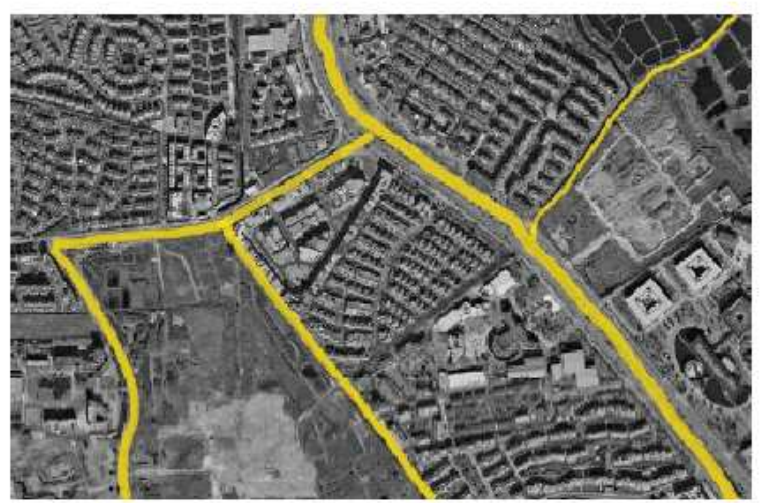

Fig. 10: The first example of road extraction results by our algorithm

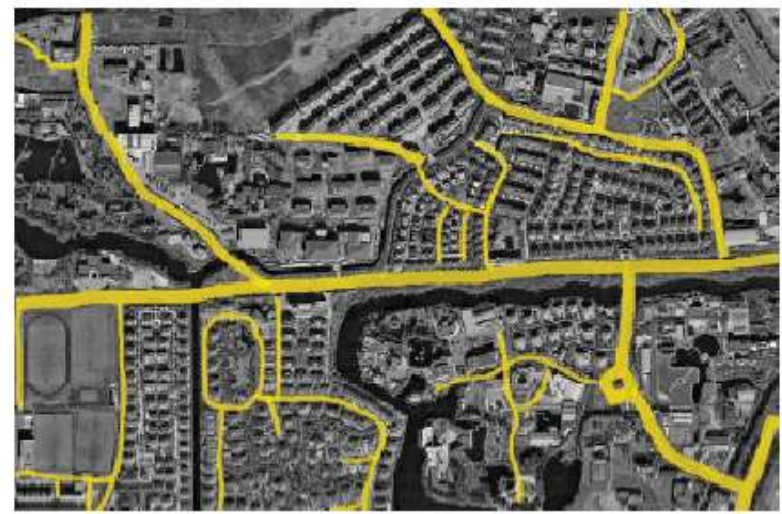

Fig. 11: The second example of road extraction results by our algorithm

\section{Conclusions and the future works}

In this paper, we present a novel road extraction algorithm based on circular projection transformation for high

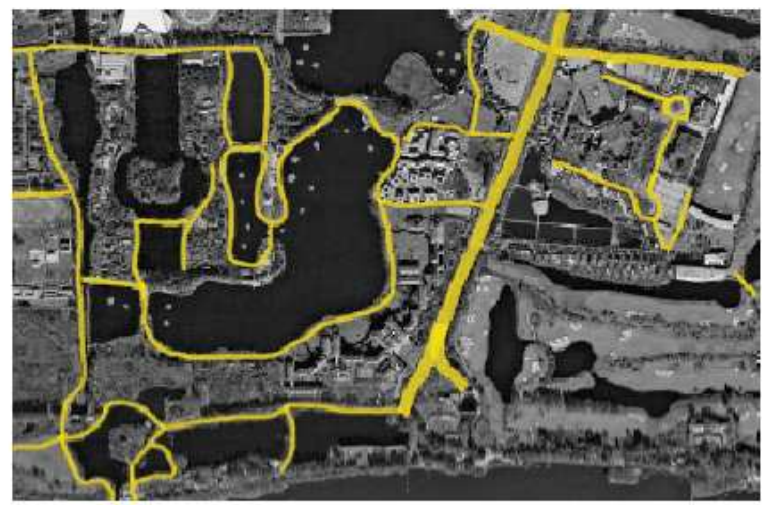

Fig. 12: The third example of road extraction results by our algorithm

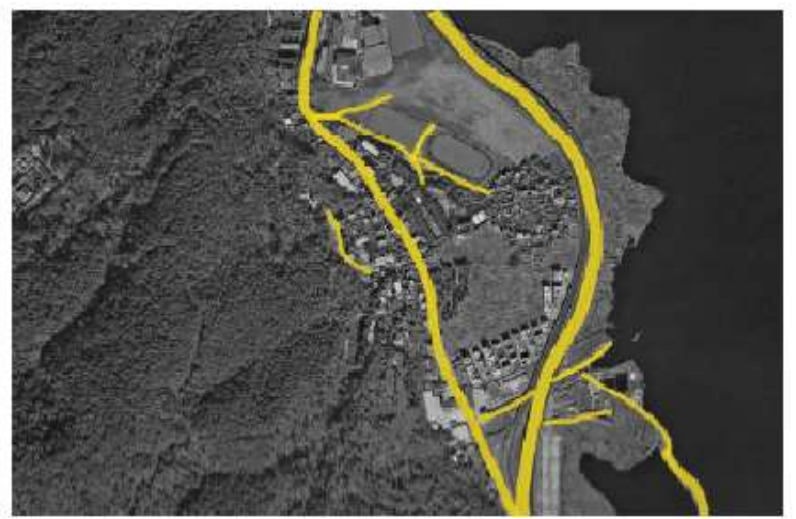

Fig. 13: The fourth example of road extraction results by our algorithm

resolution remote sensing images. In our algorithm, the road extraction results are obtained by choosing an initial template, and then search the matched templates. The circular projection vector of the initial template is achieved by calculating the circular projection value at a specific radius. After the optimal radius of the circle in circular projection transformation and the length of the seeking step and the seeking angle are obtained, roads can be detected by the correct direction by exchanging the first two detected points.

In the future works, the proposed works should be extended in the following two aspects: 1) The roads should be organized in the hierarchical mode according to the importance of each road, and 2) some other dataset should be used to make the performance evaluation more objectively. 


\section{References}

[1] Melgani F, Bruzzone L, Classification of hyperspectral remote sensing images with support vector machines, IEEE Transactions on Geoscience and Remote Sensing, 42, 17781790 (2004).

[2] Benediktsson JA, Pesaresi M, Arnason K, Classification and feature extraction for remote sensing images from urban areas based on morphological transformations, IEEE Transactions on Geoscience and Remote Sensing, 41, 1940-1949 (2003).

[3] Toutin T, Review article: Geometric processing of remote sensing images: models, algorithms and methods, International Journal of Remote Sensing, 25, 1893-1924 (2004)

[4] Bruzzone Lorenzo, Chi Mingmin, Marconcini Mattia, A novel transductive SVM for semisupervised classification of remote-sensing images, IEEE Transactions on Geoscience and Remote Sensing, 44, 3363-3373 (2006).

[5] Naixia Mou, Bing Liu, Xiushan Lu, Lingxian Zhang, Wenbao Liu, Semantic-Based Remote Sensing Images Intelligent Service on Grid Environment, In First International Workshop on Database Technology and Applications, 291294 (2009).

[6] Chaudhuri D., Kushwaha N. K., Samal A., Semi-Automated Road Detection From High Resolution Satellite Images by Directional Morphological Enhancement and Segmentation Techniques, IEEEE Journal of Selected Topics in Applied Earth Observations and Remote Sensing, 5, 1538-1544 (2012).

[7] He YH, Wang H, Zhang B, Color-based road detection in urban traffic scenes, IEEE Transactions on Intelligent Transportation Systems, 5, 309-318 (2004).

[8] Wijesoma WS, Kodagoda KRS, Balasuriya AP, Roadboundary detection and tracking using ladar sensing, IEEE Transactions on Robotics and Automation, 20, 456-464 (2004).

[9] Hu Jiuxiang, Razdan Anshuman, Femiani John C., Road network extraction and intersection detection from aerial images by tracking road footprints, IEEE Transactions on Geoscience and Remote Sensing, 45, 4144-4157 (2007)

[10] Jeon BK, Jang JH, Hong KS, Road detection in spaceborne SAR images using a genetic algorithm, IEEE Transactions on Geoscience and Remote Sensing, 40, 22-29 (2002).

[11] Tupin F, Houshmand B, Datcu M, Road detection in dense urban areas using SAR imagery and the usefulness of multiple views, IEEE Transactions on Geoscience and Remote Sensing, 40, 2405-2414 (2002).

[12] Zhang Linjuan, Gao Deyun, Zhao Weicheng, A multilevel information fusion approach for road congestion detection in VANETs, Mathematical and Computer Modelling, 58, 12061221 (2013).

[13] Jiang Haibo, Xiao Yilong, Zhang Yunwei, Curve path detection of unstructured roads for the outdoor robot navigation, Mathematical and Computer Modelling, 58, 536544 (2013).

[14] Demissie Merkebe Getachew, de Almeida Correia Goncalo Homem, Bento Carlos, Intelligent road traffic status detection system through cellular networks handover information: An exploratory study, Transportation Research Part C-emerging Technologies, 32, 76-88 (2013).
[15] Siogkas George K., Dermatas Evangelos S., RandomWalker Monocular Road Detection in Adverse Conditions Using Automated Spatiotemporal Seed Selection, IEEE Transactions on Intelligent Transportation Systems, 14, 527538 (2013)

[16] Shang Erke, An Xiangjing, Li Jian, Robust Unstructured Road Detection: The Importance of Contextual Information, International Journal of Advanced Robotic Systems, Article No. 179, 10, (2013).

[17] Oliveira Henrique, Correia Paulo Lobato, Automatic Road Crack Detection and Characterization, IEEE Transactions on Intelligent Transportation Systems, 14, 155-168 (2013)

[18] Cheng Jianghua, Jin Tian, Ku Xishu, Road junction extraction in high-resolution SAR images via morphological detection and shape identification, Remote Sensing Letters, 4, 296-305 (2013).

[19] Unsalan Cem, Sirmacek Beril, Road Network Detection Using Probabilistic and Graph Theoretical Methods, IEEE Transactions on Geoscience and Remote Sensing, 50, 44414453 (2012).

[20] Guo Chunzhao, Mita Seiichi, McAllester David, Robust Road Detection and Tracking in Challenging Scenarios Based on Markov Random Fields With Unsupervised Learning, IEEE Transactions on Intelligent Transportation Systems, 13, 1338-1354 (2012).

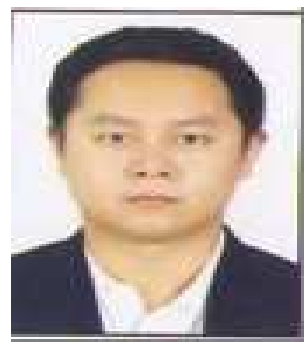

Teng Xinpeng was born in Jilin China, November, 1983. Jiangsu University of China in 2007 admitted to pursuing a doctoral computer. The main research directions: database mining and computer network communications. Mr. Teng has been keen on researching what he is interested in and devoted his most time and energy to it.

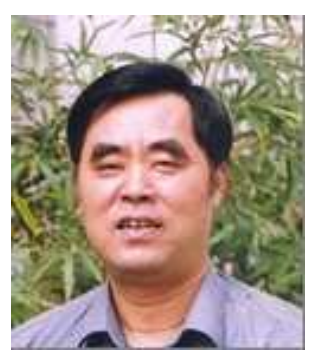

Song Shunlin was
born in August 1947, Professor of Computer Science, doctoral tutor. The Jiangsu University Librarian; Members CG / CAD Professional Committee of China Computer Federation; Jiangsu Province Computer Society; learn computer Zhenjiang City chairman; pay Zhenjiang City Library Association chairman; Jiangsu Province, enterprise information technology consultant. 1970, Nanjing University mathematics graduate, 1978.9 $\sim 1980.2$ in Nanjing University, Department of Computer Science Education, $1991.10 \sim 1993.4$ Japan Faculty of Engineering, Information Engineering disciplines visiting scholar at Mie University, $1998.12 \sim 1999.5$ Japan Faculty of Engineering Systems discipline senior visiting scholar at Osaka University in. 


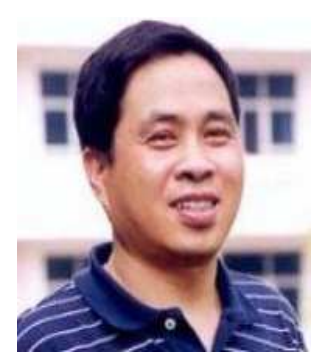

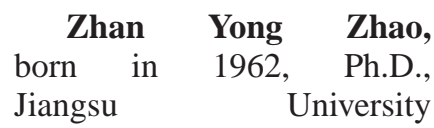
professor. Mainly engaged in distributed systems, multimedia technology, pattern recognition and the direction of teaching and research work. Presided over the completion of the main participants or 863 high-tech

projects, the National Natural Science Foundation of China and the provincial and ministerial research 9, provincial, ministerial level scientific and technological progress awards 4. Edited three academic books, academic journals and conferences at home and abroad published more than 40 papers, which included 7, ISTP included 2. 2002 was named the province "Blue Project" training candidates. Each one is responsible for the National Natural Science Foundation and the Natural Science Foundation of provincial universities, a number of horizontal cooperation projects. 